\title{
POSTER 27-92
}

\section{Prehospital Care Providers Do Not Accurately Identify Motor-Vehicle Crash Patients with Positive Serum Alcohol Concentrations: A Brief Report}

\author{
Ronald F. Maio, Do, ${ }^{1}{ }^{*}$ Audrey $W u, B S,{ }^{2}$ Frederic \\ C. Blow, PhD, ${ }^{3}$ Brian J. Zink, $\mathrm{MD}^{4}$ \\ 1. Section of Emergency Medicine, Department of Surgery, \\ University of Michigan, Ann Arbor, Michigan \\ 2. Medical Student, University of Michigan, Ann Arbor, \\ Michigan \\ 3. Alcohol Research Center, University of Michigan \\ 4. Section of Emergency Medicine, Department of Surgery, \\ University of Michigan, Ann Arbor, Michigan
}

Introduction: Alcohol use is associated with many motorvehicle crashes (MVC), may increase the severity of injury, and may also mask in MVC resulting in inappropriate assessment by prehospital care providers (PHCP). To appropriately assess and triage MVC, victims' PHCPs must accurately identify recent alcohol use.

Objective: To examine the accuracy of PHCPs in identifying MVC victims with positive serum alcohol concentrations (SAC+).

Design: Retrospective cohort study.

Population: MVC victims $>=17$ years of age, brought directly to the Emergency Department by ground ambulance $(\mathrm{n}=372)$.

Time Period: 15 July 1990 to 15 July 1991

Methods: Data sources were ambulance report forms (ARP) and hospital records. Variables abstracted included Revised Trauma Score (RTS), PHCP's impression of alcohol use and SACs. Sensitivity (SENS), specificity (SPEC), positive predictive value (PPV), negative predictive value (NPV), and 0.95 confidence intervals $(0.95 \mathrm{CI})$ were calculated for PHCP's ability to identify patients with a SAC $>0$. The relationship between RTS and the impressions of alcohol use was analyzed using Chi-square testing: a $p$-value of $<0.05$ was considered statistically significant.

Results: One-hundred sixty-two patients had a SAC test performed. Sensitivity was $0.39(0.28-0.51)$; SPEC 1.00 (1.00-1.00); PPV 1.00 (1.00-1.00); NPV 0.69 (0.58-0.80). The relationship between injury severity (RTS) and impressions of alcohol use was not statistically significant.

Conclusion: This study suggests that prehospital care providers cannot separately identify SAC+ MVC victims: selection bias and retrospective design are limitations of this study. Future studies should evaluate methods to improve PHCP's accuracy in assessing alcohol use. 